\title{
Effects and Interferences of Emicizumab, a Humanised Bispecific Antibody Mimicking Activated Factor VIII Cofactor Function, on Coagulation Assays
}

\author{
Joanne I. Adamkewicz ${ }^{1}$ David C. Chen ${ }^{1} \quad$ Ido Paz-Priel $^{1}$ \\ ${ }^{1}$ Genentech, Inc., South San Francisco, California, United States \\ Address for correspondence Joanne I. Adamkewicz, PhD, Genentech, \\ Inc., 1 DNA Way, MS 422a, South San Francisco, CA 94080, \\ Thromb Haemost 2019;119:1084-1093. \\ United States (e-mail: adamkewicz.joanne@gene.com).
}

\begin{abstract}
Emicizumab bridges activated factor IX (FIX) and FX to restore the tenase function mediated by activated FVIII (FVIIIa), which is deficient in people with haemophilia A (PwHA). Unlike FVIII, emicizumab does not require activation to function; thus, in coagulation assays, the behavior of emicizumab may differ from that of FVIII. The objective of this study was to assess the effect of emicizumab on coagulation assays, including potential interference behavior that may produce inaccurate or misleading results. A variety of clotting-based, amidolytic/ chromogenic, latex particle-enhanced turbidometric, and enzyme-linked immunosorbent methods were investigated. As expected based on its pharmacologic mechanism of action, emicizumab exhibited strong activity on the activated partial thromboplastin time (aPTT), which resulted in interference with several aPTT-based assays, most importantly the onestage FVIII activity assay; these assays are not recommended for PwHA receiving emicizumab therapy. Pharmacodynamic activity of emicizumab, as measured by FVIII chromogenic

Keywords

- emicizumab

- haemophilia A

- blood coagulation factors

- coagulation assays

- interference assays, was species-dependent due to the binding specificity of the drug antibody. Outside of FVIII assays, emicizumab did not interfere with assays based on immunologic or chromogenic principles, nor with clotting assays based on nonintrinsic pathway activators, thus offering alternative choices where aPTT-based assays might otherwise be used. The observed interferences are in line with the unique mechanism of action of emicizumab. Potential interferences should be taken into account in the selection of coagulation assays and interpretation of coagulation assay test results for PwHA receiving emicizumab therapy.
\end{abstract}

\section{Introduction}

Emicizumab (HEMLIBRA; F. Hoffmann-La Roche, Basel, Switzerland) is a recombinant, humanised, bispecific monoclonal antibody that bridges activated factor (F) IX and FX to restore the function of missing activated FVIII (FVIIIa) in people with haemophilia $\mathrm{A}$ (PwHA). ${ }^{1-3}$ In a multicentre, phase III trial conducted in adolescents and adults with haemophilia A with FVIII inhibitors, once-weekly administration of subcutaneous emicizumab prophylaxis reduced bleeding rates by 87\% compared with no prophylaxis. ${ }^{4}$ Emicizumab was also efficacious when administered to PwHA with FVIII inhibitors under the age of 12 in an additional phase III trial. ${ }^{5}$ Further

received

October 15, 2018

accepted after revision

March 19, 2019
DOI https://doi.org/ $10.1055 / \mathrm{s}-0039-1688687$. ISSN 0340-6245. studies demonstrated superior efficacy of emicizumab compared with FVIII prophylaxis for the control of bleeding in PwHA without inhibitors, ${ }^{6}$ and consistent efficacy was shown when administered once-weekly, every 2 weeks, or every 4 weeks in PwHA with or without inhibitors. ${ }^{6,7}$ Following the outcomes of these trials, emicizumab administered subcutaneously once-weekly, every 2 weeks, or every 4 weeks has been approved in several countries (including the United States, European Union member states, Australia, and Japan) as a prophylactic therapy for PwHA of all age groups with or without FVIII inhibitors.

Integrating a novel therapeutic agent like emicizumab into clinical practice requires changes in laboratory practice @ 2019 Georg Thieme Verlag KG
Stuttgart · New York
License terms

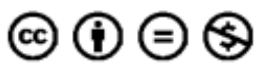


that are appropriate for the new mechanism of action. FVIII activation is a rate-limiting step in the coagulation cascade ${ }^{8}$; but unlike FVIII, emicizumab does not require activation to perform its function ( - Fig. 1). ${ }^{3}$ Therefore, the interactions of emicizumab with coagulation assays are expected to differ from those of FVIII. For example, emicizumab has been shown to have a strong procoagulant effect on activated partial thromboplastin time (aPTT).$^{9-11}$ As such, adjustments in clinical practice with regard to assay selection or laboratory test result interpretation, or both, are required for the appropriate management of PwHA treated with emicizumab. ${ }^{12,13}$

The objective of this exploratory study was to profile the effect of emicizumab on a wide range of in vitro coagulation assays commonly used in clinical practice. A variety of analytes and methodologies were investigated to inform a systemic understanding of emicizumab activity and interference effects, while emphasizing assays routinely used in haemophilia Aincluding aPTT, prothrombin time (PT), and FVIII activity. Surveyed methodologies included assays triggered at different levels of the coagulation cascade and those that employed disparate measurement principles (e.g., clotting-based, amidolytic [chromogenic], latex particle-enhanced turbidometric, and enzyme-linked immunosorbent assay [ELISA] methods). Assays were run in the absence and presence of emicizumab, using a concentration range $(0-200 \mu \mathrm{g} / \mathrm{mL})$ wider than the steady-state trough plasma levels $(\sim 25-80 \mu \mathrm{g} / \mathrm{mL})$ identified for the approved weekly regimen of $3 \mathrm{mg} / \mathrm{kg}$ of body weight for 4 weeks followed by $1.5 \mathrm{mg} / \mathrm{kg}$. 4,14

\section{Materials and Methods}

\section{Single-Factor Assays}

Frozen normal pooled plasma samples consisting of plateletpoor plasma from 20 or more screened donors were sourced (CRYOcheck; Precision Biologic Inc: Dartmouth, NS, Canada). To test samples containing endogenous clotting factors at defined concentrations ( 100,50 , and $25 \%$ ), samples were either undiluted, diluted twofold, or diluted fourfold in plasma that was deficient for specific factors-either FII, FV, FVII, FVIII, FIX, FX, FXI, or FXII (Haematologic Technologies Inc., Essex Junction, Vermont, United States). Emicizumab was spiked into the plasma samples as described below.

The effect of emicizumab on single-factor activities was evaluated using one-stage PT-based (FII, FV, FVII, and FX) or aPTT-based (FIX, FXI, and FXII) assays, as well as the chromogenic FIX assay. Assay details, including reagent suppliers and coagulation instruments used, are listed in - Supplementary Table S1 (available in the online version).

\section{FVIII One-Stage and Chromogenic Assays}

Frozen plasma samples from one person with severe haemophilia A without FVIII inhibitors were sourced (George King Bio-Medical Inc., Overland Park, Kansas, United States). Plasma was spiked with emicizumab as described below.

Two chromogenic FVIII assays-one containing bovine FIXa and FX reagents and the other containing human FIXa and FX-were performed as outlined in - Table 1. The effect of emicizumab on FVIII activity was evaluated using a one-

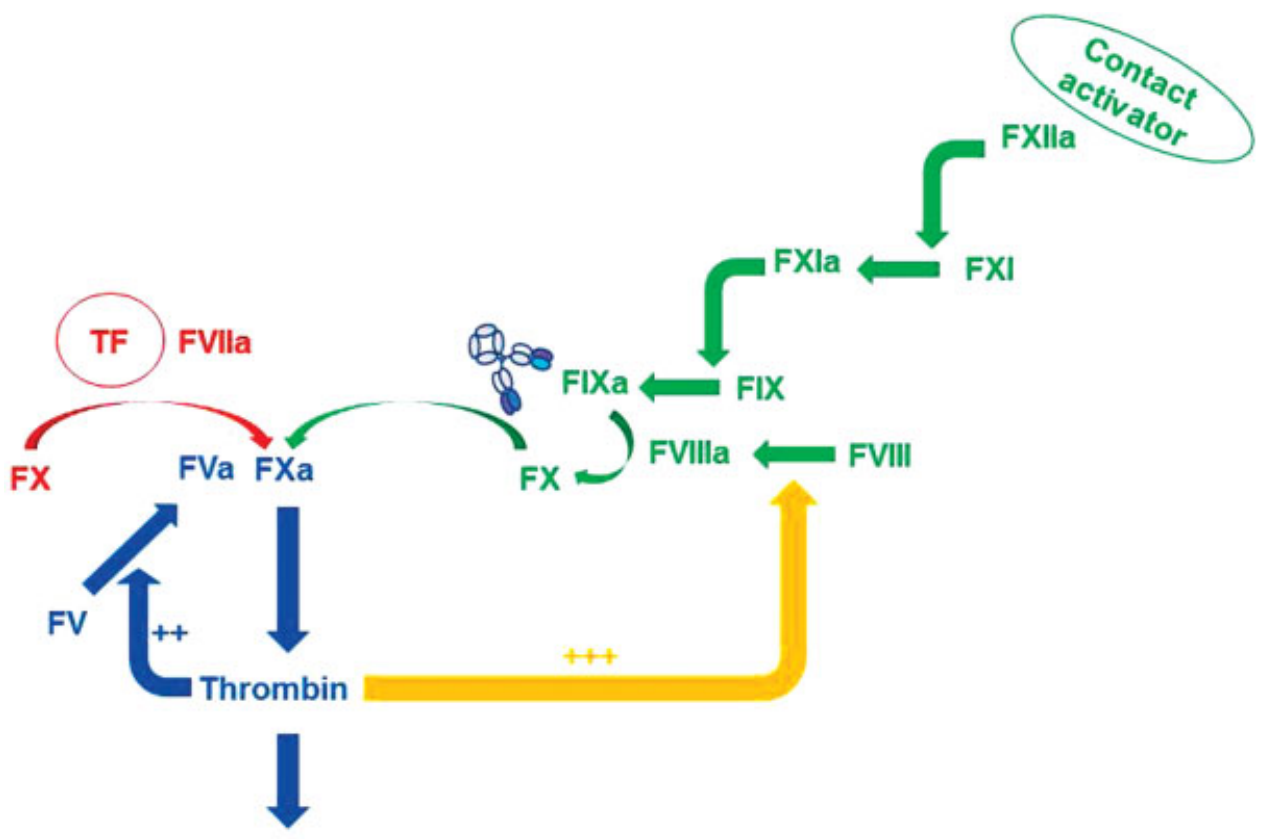

Fibrin formation

Fig. 1 Schematic representation of the level of emicizumab action with respect to the activation pathways (circles) applied for PT assays (TF) and aPTT assays (contact activator). The extrinsic pathway is represented in red, the intrinsic pathway in green, and the common pathway in blue. Thrombin propagates the coagulation cascade through a feed-forward cycle (arrows to factor V [FV]/FVa and FVIII/FVIIIa steps), leading to clot formation. The activation of FVIII (yellow arrow) is a rate-limiting step in normal haemostasis. Emicizumab, however, does not require activation for its activity, and thus propagates the coagulation cascade without the additional time needed for feed-forward activation of FVIII. Therefore, emicizumab is expected to behave differently to FVIII with respect to time to clot formation in coagulation assays. aPTT, activated partial thromboplastin time; PT, prothrombin time; TF, tissue factor. 
Table 1 FVIII one-stage and chromogenic assays performed in this study

\begin{tabular}{|l|l|l|l|l|}
\hline Analyte/assay & Kit & Assay type & Instrument & Assay details \\
\hline $\begin{array}{l}\text { aPTT-based FVIII } \\
\text { one-stage assay }\end{array}$ & $\begin{array}{l}\text { Synth-A-Sil } \\
\text { (Illinois, Bedford, United States) }\end{array}$ & Clotting & ACL TOP & $\begin{array}{l}\text { Single-factor activity is determined by } \\
\text { performing aPTT using the test sample } \\
\text { diluted in plasma deficient in the } \\
\text { specific factor being assayed }\end{array}$ \\
\hline $\begin{array}{l}\text { FVIII chromogenic, } \\
\text { bovine factors }\end{array}$ & $\begin{array}{l}\text { FVIII Chromogenic Assay } \\
\text { (Siemens, Marburg, Germany) }\end{array}$ & $\begin{array}{l}\text { Amidolytic/ } \\
\text { chromogenic }\end{array}$ & $\begin{array}{l}\text { BCS XP } \\
\text { (Siemens) }\end{array}$ & $\begin{array}{l}\text { Purified FIXa and FX proteins, calcium, } \\
\text { phospholipids, and a synthetic substrate } \\
\text { for FXa are mixed with sample; color } \\
\text { development is proportional to the } \\
\text { tenase cofactor activity in the sample } \\
\text { human factors } \\
\text { (FVIIIla or emicizumab), which is rate } \\
\text { limiting in the presence of excess FIXa }\end{array}$ \\
\hline
\end{tabular}

Abbreviations: ACL TOP, ACL TOP 500 CTS analyser (IL); aPTT, activated partial thromboplastin time; BCS XP, BCS XP Analyzer; IL, Instrumentation Laboratory; STA-R, STA-R Evolution analyser.

stage FVIII assay ( - Table 1). Samples with reported activities that exceeded the calibration range (150\%) were diluted 10 fold in saline and reanalysed.

\section{Additional Coagulation Assays}

Coagulation assays for global clotting (aPTT, PT, and thrombin time), protein $C$, protein $S$, activated protein $C$ (APC) resistance, fibrinogen, von Willebrand factor ( $\mathrm{VWF}$ ) antigen and activity, plasminogen antigen and activity, antithrombin, D-dimer, FXIII antigen, and anti-Xa activity were performed using commercially available diagnostic kits according to instructions provided by the manufacturers (-Supplementary Table S1, available in the online version). Some kits included human plasma as part of the test method.

Plasma samples from PwHA without inhibitors $(n=3)$, healthy volunteers (i.e., controls; $n=3$ ), persons without haemophilia A treated with vitamin $\mathrm{K}$ antagonists (VKAs) resulting in elevated international normalised ratio (INR; $n=3$ ), and one individual with elevated D-dimer concentration were sourced (Biomex, Heidelberg, Germany).

All samples were collected using standard venipuncture blood collection tubes containing one-tenth volume sodium citrate $(3.2 \% / 0.109 \mathrm{M})$ to give a final citrate concentration of $0.32 \% / 0.0109 \mathrm{M}$. Plasma was separated by centrifugation and frozen at $-70^{\circ} \mathrm{C}$ until the time of analysis. After thawing, the plasma samples were differentially spiked with emicizumab as described below. aPTT and anti-Xa activity were also tested in samples that were spiked with 0.5 and $1 \mathrm{U} / \mathrm{mL}$ unfractionated heparin (UFH) solution (heparin sodium BRP, European Directorate for the Quality of Medicines, Strasbourg, France). The sample with elevated D-dimer concentration was analyzed undiluted and following twofold and fourfold dilution in normal control plasma to create three samples with elevated D-dimer values.

\section{Plasma Spiking with Emicizumab}

Working stocks of emicizumab (Chugai Pharmaceutical Co. Ltd., Tokyo, Japan) were made fresh daily in Tris-buffered saline $\mathrm{pH}$ 7.6. Thawed plasma samples were spiked with emicizumab working stocks at a final concentration of $1 \%$ (vol/vol); for example, $5 \mu \mathrm{L}$ of emicizumab (or control buffer) was added to $495 \mu \mathrm{L}$ plasma, to produce final plasma con- centrations ranging from 0 (control) to $200 \mu \mathrm{g} / \mathrm{mL}$, which ranged above and below the reported phase III steady-state trough plasma concentration range of approximately 25 to $80 \mu \mathrm{g} / \mathrm{mL} .{ }^{4}$ A plasma concentration of $50 \mu \mathrm{g} / \mathrm{mL}$ corresponds to the median expected clinical trough concentration of emicizumab, while a concentration of $200 \mu \mathrm{g} / \mathrm{mL}$ corresponds to a supratherapeutic drug level.

\section{Data Analyses}

Ethical committee approval was obtained for the commercial plasma sampling by the applicable vendors, including George King, Biomex, and Haematologic Technologies. All analyses were performed in duplicate and the results are reported as means of the two determinations. The samples assayed for each experiment are specified in the figure legends.

\section{Results}

\section{Global Coagulation Cascade Assays}

Global assays of coagulation utilizing either intrinsic, extrinsic, or common pathway triggering reagents were investigated. Emicizumab shortened the aPTT values of both haemophilia A and control plasma samples ( - Fig. 2A); aPTT values decreased dramatically at the lowest tested emicizumab concentration, $10 \mu \mathrm{g} / \mathrm{mL}$, and reached near-maximal reduction at $50 \mu \mathrm{g} / \mathrm{mL}$. This is consistent with previous reports. ${ }^{9-11,15}$ In contrast, emicizumab had no effect on the thrombin time in either haemophilia A or control samples (-Fig. 2B), indicating that emicizumab does not interfere with coagulation activated at the level of the common pathway.

The effects of emicizumab on PT were nuanced and reagentdependent. A measurable increase in INR was observed using four different reagents when increasing concentrations of emicizumab were added to plasma samples from healthy donors (-Fig. 3A-D, dashed lines). However, the average increase in INR with emicizumab $100 \mu \mathrm{g} / \mathrm{mL}$ versus unspiked samples was extremely small (between 0.01 and 0.05 across reagents). Although anticoagulation therapy is not a common clinical scenario for PwHA, to further characterise the effect of emicizumab on INR we examined plasma samples from donors without haemophilia A who were being treated with VKA. From starting INR values between 2 and 5, spiking with 

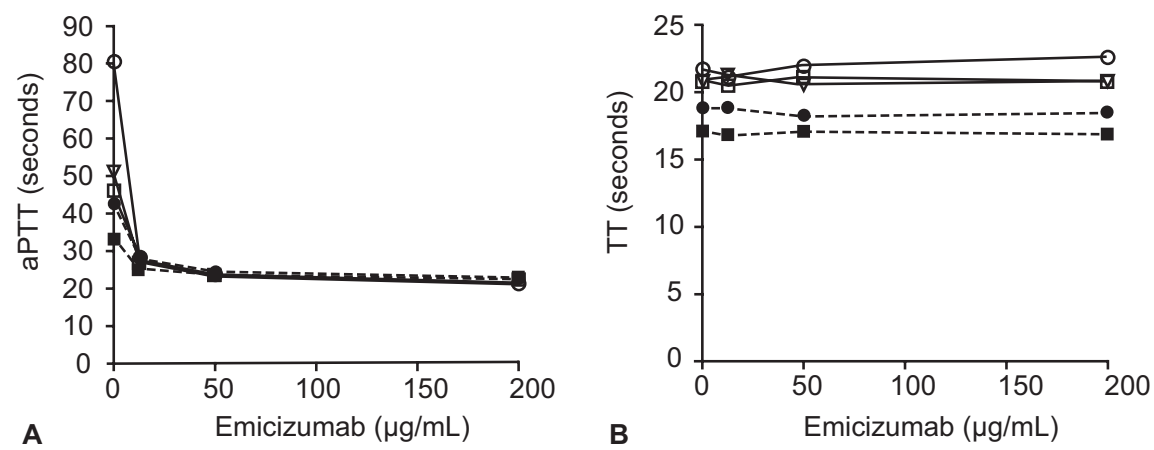

Fig. 2 The effect of emicizumab on aPTT and thrombin time. aPTT (A) and thrombin time (B) were determined in three haemophilia A samples (solid lines, open symbols) and two control normal plasma samples (dashed lines, closed symbols), which were spiked with increasing concentrations of emicizumab. aPTT, activated partial thromboplastin time; TT, thrombin time.
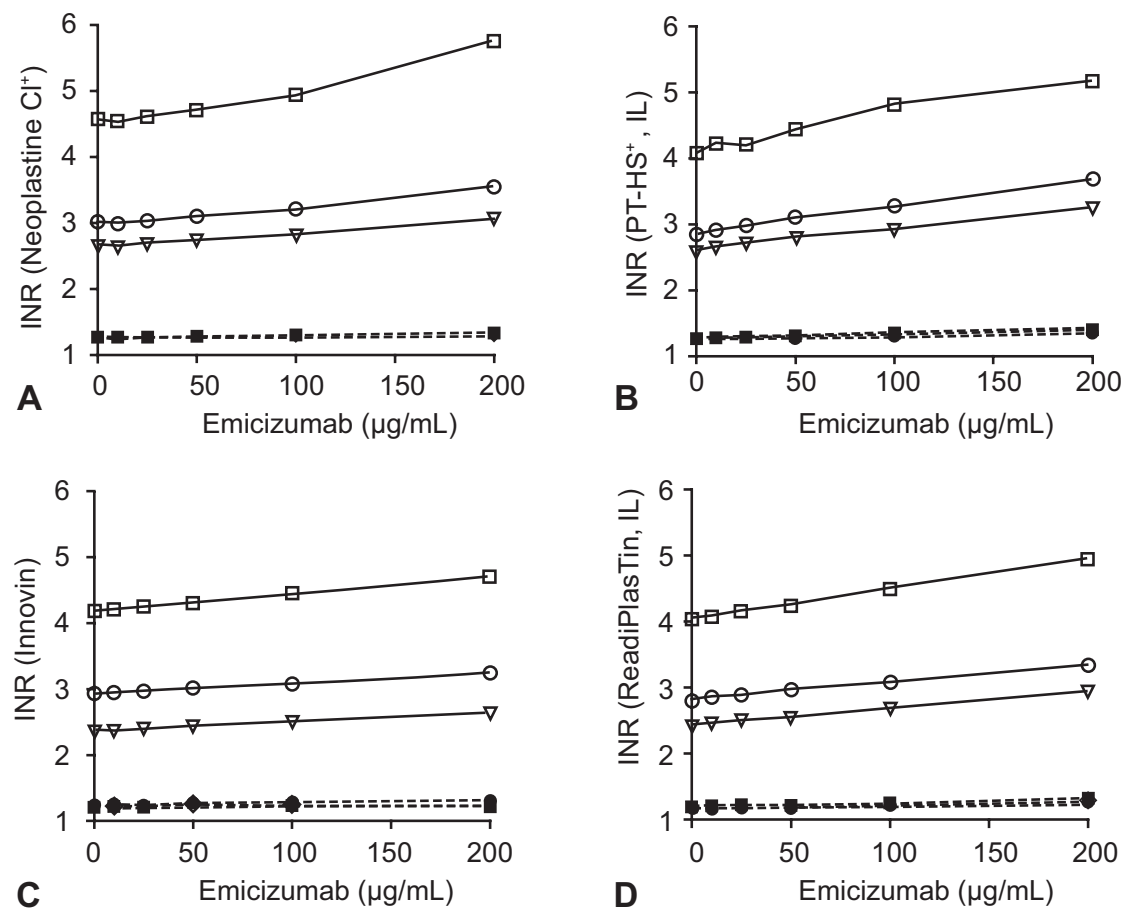

Fig. 3 The effect of emicizumab on INR. PT/INR was measured using four different reagents: (A) Neoplastine $\mathrm{Cl}^{+}$, (B) PT-HS $\mathrm{H}^{+}$, (C) Innovin, and (D) ReadiPlasTin. Three samples from persons receiving vitamin $\mathrm{K}$ antagonists (solid lines, open symbols) and three control plasma samples (dashed lines, closed symbols) were analyzed. Emicizumab was added, at indicated concentrations, to each sample and the average of two determinations is presented. HS, high sensitivity; IL, Instrumentation Laboratory; INR, international normalised ratio; PT, prothrombin time.

emicizumab led to an increase in INR between 0.18 and 0.50 across reagents (-Fig. 3A-D, solid lines; - Supplementary Table S2, available in the online version).

\section{FVIII Assays}

The most prevalent method of measuring FVIII activity is the so-called "one-stage" method, which relies on correction of the aPTT-based clotting time of FVIII-deficient plasma. ${ }^{16}$ As expected based on assay principles, the dramatic shortening of aPTT caused by emicizumab resulted in strong interference with the one-stage FVIII activity assay, leading to out-of-range values exceeding $150 \%$ reported FVIII activity, even at very low emicizumab concentrations (data not shown). In many coagulation laboratories, out-of-range values for factor assays result in reflex testing of samples at higher dilutions, ${ }^{17}$ so to assess the potential implications of emicizumab interference in this scenario, we diluted FVIII-deficient, emicizumab-spiked plasma in saline prior to measurement to bring the reported result into the analytical measuring range of the assay. In this experiment, emicizumab that was spiked into FVIII-deficient plasma showed 8 to $12 \%$ FVIII activity per $\mu \mathrm{g} / \mathrm{mL}$ emicizumab in the one-stage assay ( - Fig. 4A). Thus, the dilution-corrected FVIII activity values reported for clinically relevant concentrations of emicizumab were well in excess of $250 \%$.

Chromogenic assays for FVIII activity are also available in clinical practice. ${ }^{18}$ These assays isolate the factor-specific enzymatic reaction being tested. We measured the pharmacodynamic effect of emicizumab on two commercially available FVIII chromogenic assays manufactured using bovine or human FIXa and FX reagents in the test kits. As expected 

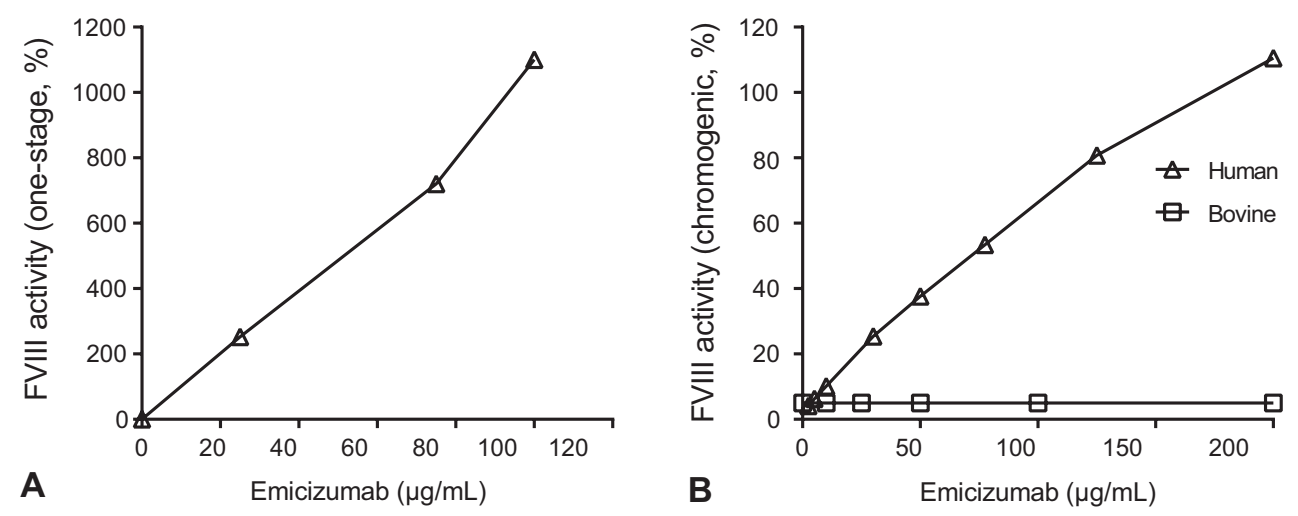

Fig. 4 Effect of emicizumab on factor VIII (FVIII) activity. FVIII-deficient plasma was spiked with increasing emicizumab concentrations. (A) FVIII activity was determined by an aPTT-based one-stage FVIII assay. Samples containing emicizumab exceeded the calibration range (150\%) were diluted in saline and measured again. Averages of FVIII results from two independent determinations, corrected for dilution, are presented. (B) FVIII concentrations were assessed using two chromogenic FVIII assays using human or bovine FX and FIXa. aPTT, activated partial thromboplastin time.

from its species specificity, emicizumab exhibited no FVIII activity at tested concentrations in the assay containing bovine FX and FIXa. However, a concentration-dependent effect of emicizumab on FVIII activity readout was observed in the assay containing human FIXa and FX (-Fig. 4B).

\section{Single-Factor Assays: Intrinsic Pathway}

The effects of emicizumab on additional tests using onestage or alternative methodologies were explored. We evaluated aPTT-based one-stage assays for FIX, FXI, and FXII in the absence or presence of emicizumab. Even at the lowest emicizumab concentration tested $(10 \mu \mathrm{g} / \mathrm{mL}$; well below the clinical range), a twofold increase in factor activity levels were reported compared with samples without emicizumab (-Fig. 5A-C). Importantly, in a chromogenic methodology, emicizumab showed no effect on FIX activity (-Fig. 5D).

Similarly, increasing concentrations of emicizumab resulted in pronounced dose-dependent reductions of reported protein $\mathrm{C}$ and protein $\mathrm{S}$ activities when measured using aPTT-based assays: their reported activities were reduced by approximately twofold at clinically relevant emicizumab concentrations (-Supplementary Fig. S1A and S1B, available in the online version). In contrast, emicizumab had no effect on the chromogenic protein $C$ assay or the immunologic free protein $S$ antigen assay (- Supplementary Fig. S1C and S1D, available in the online version). Emicizumab also significantly reduced the APC resistance ratio in FV-deficient plasma using the aPTT-based Coatest APC resistance assay ( - Supplementary Fig. S2A, available in the online version) and yet exhibited no effect on the APC resistance ratio using the prothrombin activator-based Acticlot protein $C$ resistance assay, despite some analytical variability in samples with high ratios (- Supplementary Fig. S2B, available in the online version). Finally, an assessment was performed using two control (healthy donor) samples spiked with UFH. Even in the presence of UFH, emicizumab shortened the aPTT values in a concentration-dependent manner ( - Fig. 6A, B), yet had no effect on an alternative assay that measures production of a defined chromogenic substrate, the anti-Xa activity, with or without spiked UFH (-Fig. 6C, D).
Single-Factor Assays: Common and Extrinsic Pathways We determined whether the smaller perturbation of emicizumab on INR would be observed in assays that rely on the PT. We evaluated the effect of emicizumab on the reported activities of FII, FV, FVII, and FX, as determined by the respective PT-based one-stage single-factor assays. Plasma samples spiked with emicizumab demonstrated stable activity levels for FII, FV, FVII, and FX (- Supplementary Fig. S3, available in the online version). Derived fibrinogen levels were calculated from the change in optical density during the PT test. The effect of emicizumab on the reported INR was expected to carry over to the derived fibrinogen concentration; indeed, weak but detectable reductions in derived fibrinogen levels (6-17\%) were reported with increasing concentrations of emicizumab $(50-200 \mu \mathrm{g} / \mathrm{mL})$ compared with samples without emicizumab (-Supplementary Fig. S4A, available in the online version). Emicizumab had no effect on fibrinogen concentrations determined using the Clauss method ${ }^{19}$ ( - Supplementary Fig. S4B, available in the online version).

In a further survey of assays using immunogenic or chromogenic methods, rather than clotting time-based methods, emicizumab had no detectable effect on measurements of multiple coagulation proteins: vWF antigen, vWF activity, FXIII antigen concentration, antithrombin activity, plasminogen activity, plasminogen antigen, and D-dimer (-Supplementary Fig. 55, available in the online version).

\section{Discussion}

In this study, we assessed the effects of emicizumab on a wide variety of coagulation assays, to guide how clinical laboratory practices may require adaptation. Based on our survey utilizing spiked plasma samples, common coagulation assays may be divided into three categories reflecting the extent to which they are affected by clinically relevant concentrations of emicizumab $(\sim 25-80 \mu \mathrm{g} / \mathrm{mL}){ }^{4}{ }^{4}$ The first group were those unaffected by emicizumab (no interference); the second, those in which the small but measurable effect of emicizumab does not impact clinical utility or interpretation of results (weak interference); and the third, those assays that are strongly 

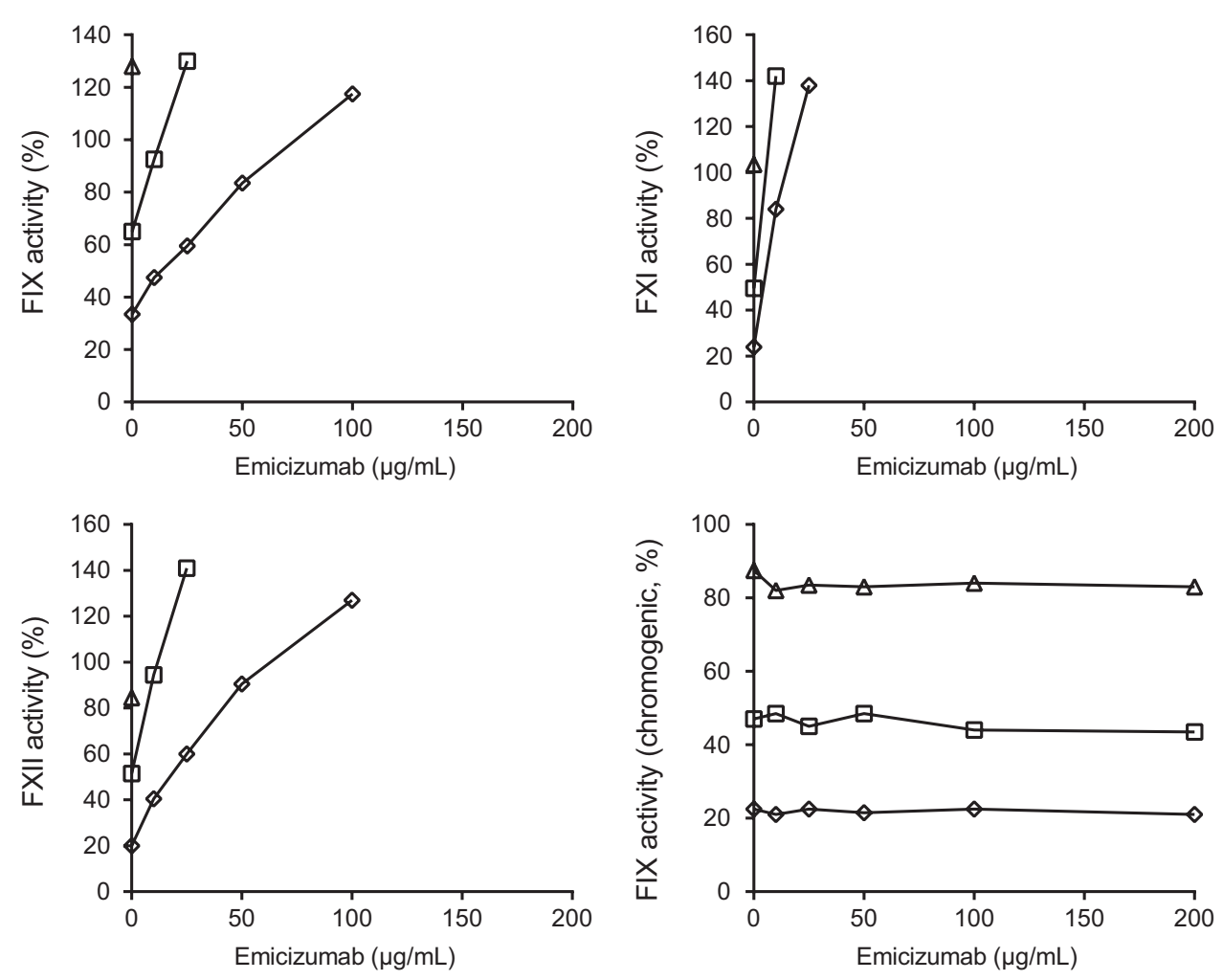

Fig. 5 Effect of emicizumab on the activities of (A) factor IX (FIX), (B) FXI, and (C) FXII as determined by the aPTT-based one-stage assays, and (D) on FIX activity as determined by the chromogenic FIX assay. The diamonds represent the undiluted plasma pool, the squares a twofold dilution in plasma deficient for the corresponding factor, and the triangles a fourfold dilution. Missing data points indicate that the result was $>150 \%$. aPTT, activated partial thromboplastin time.

affected, and whose correct interpretation requires knowledge that the sample contains emicizumab (strong interference). This third category includes human FVIII chromogenic assays, which exhibit a dose-response relationship to emicizumab concentration, as expected from a pharmacodynamic assay; however, such activity constitutes interference when attempting to measure endogenous or infused FVIII activity in the presence of emicizumab.

Assays in which no interference by emicizumab was detected were those using chromogenic (e.g., protein C, antithrombin, and anti-Xa), immunogenic ELISA-based (e.g., plasminogen), or latex particle-enhanced turbidometry methods (e.g., free protein S, D-dimer, vWF antigen, vWF activity, FXIII antigen). Emicizumab also had no detectable effect on clotting assays triggered by thrombin or FXa (i.e., thrombin time or fibrinogen) or by a prothrombin activator. In assays that did not show interference, the lack of interference was due to the underlying principles of these assays, which are unaffected by the biochemical properties of emicizumab. For instance, the chromogenic FIX kit contains FVIII that is activated by thrombin prior to the detection of FIX activity in the sample, such that FVIIIa is not limiting. In this case, emicizumab is not required to provide cofactor activity for the tenase reaction. The lack of emicizumab interference in the chromogenic FIX assay supports the hypothesis that the high activity of emicizumab in time-based assays is due to the fact that emicizumab -unlike FVIII-does not require activation, a typically ratelimiting step that contributes to total clotting time. ${ }^{3}$
Assays in which weak interference by emicizumab were detected included the PT and PT-derived fibrinogen assays, which are not triggered by the intrinsic pathways but in which FXa is generated during the reaction. The influence of emicizumab was reflected in the INR and was more pronounced when using the PT reagent $\mathrm{PT}-\mathrm{HS}^{+}$vs ReadiPlasTin, Neoplastine $\mathrm{CI}^{+}$, or Innovin; similar reagent-dependent effects have been seen with other drugs such as telavancin. ${ }^{20}$ In plasma samples from normal donors as well as those from donors receiving VKA therapy, emicizumab increased INR by minor increments that are too small to indicate increased bleeding risk ${ }^{21-23}$ and hence are unlikely to be of clinical relevance. Emicizumab also had a small and clinically insignificant effect on the measurement of derived fibrinogen, which was calculated from parameters measured during the PT assay. These weak effects may be due to steric interferences related to the binding of emicizumab to FX/FXa. ${ }^{3}$

Emicizumab demonstrated strong interference in clottingbased assays that use an intrinsic pathway trigger (i.e., aPTT and one-stage assays based on aPTT). These included aPTTbased assays for FVIII, FIX, FXI, FXII, protein C, or protein S activity, as well as the aPTT-based APC resistance assay. To highlight the extent of this interference, in FVIII-depleted plasma, 250\% FVIII activity was reported for a sample with an emicizumab concentration of $25 \mu \mathrm{g} / \mathrm{mL}$ (at the low end of the clinical range). ${ }^{4}$ The reported FVIII activities markedly overestimate the coagulation potential of emicizumab, as 

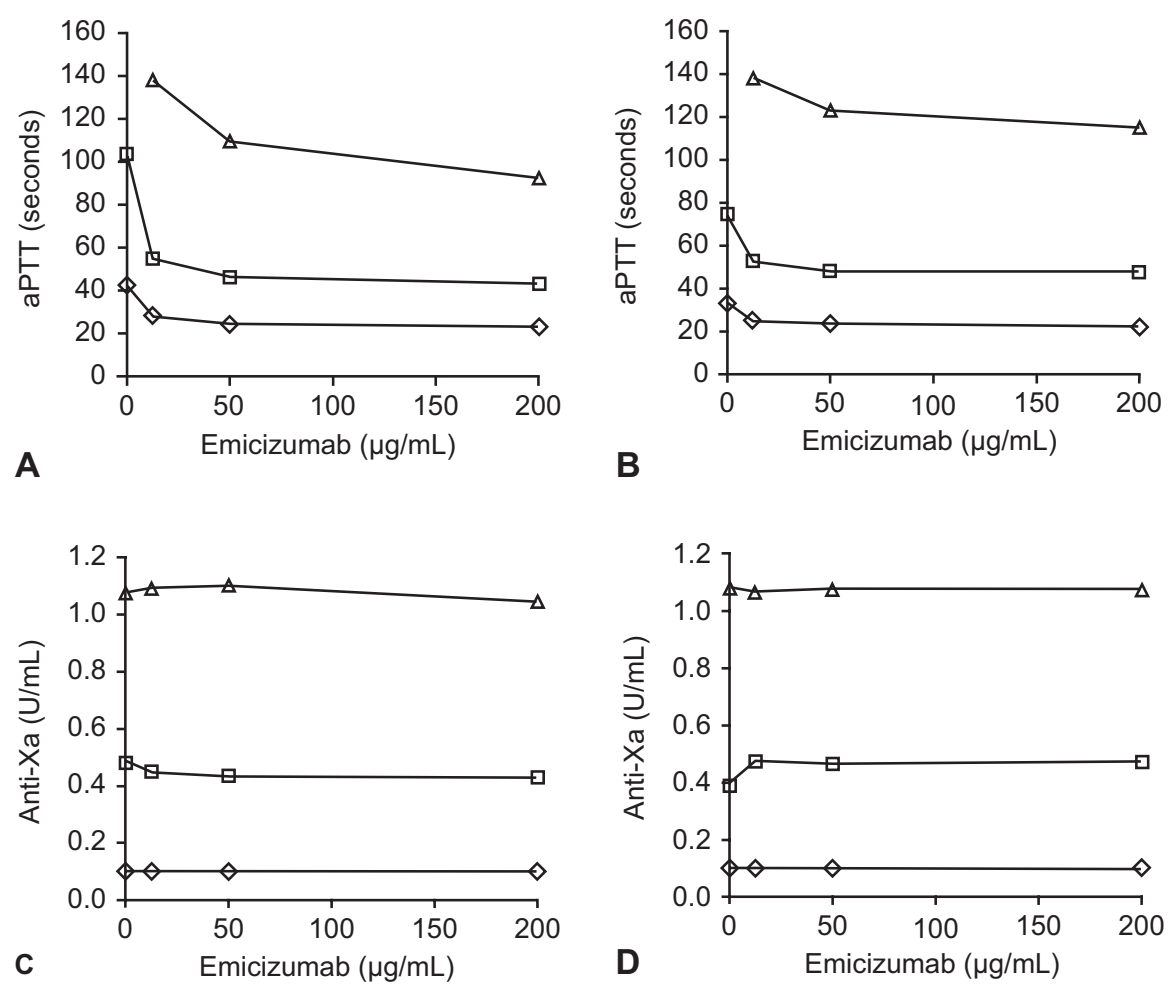

Fig. 6 The effect of emicizumab on aPTT and anti-Xa activity. Control plasma samples from two healthy donors were tested without UFH (diamonds), spiked with $0.5 \mathrm{U} \mathrm{UFH} / \mathrm{mL}$ (squares), and spiked with $1 \mathrm{U} \mathrm{UFH} / \mathrm{mL}$ (triangles). (A, C) Results of one spiked normal plasma sample. (B, D) Other sample. aPTT, activated partial thromboplastin time; UFH, unfractionated heparin.

suggested by substantially reduced but not eliminated bleed rates in clinical trials. ${ }^{4,6,10}$

Emicizumab also shortened the aPTT in plasma samples containing UFH. Although not tested here, it is probable that emicizumab would interfere with the activated clotting time (ACT), due to the similar principles of the assays used to assess $\mathrm{ACT}^{24}$ and aPTT. This prediction regarding ACT should be confirmed experimentally. In contrast, emicizumab did not affect measurement of chromogenic anti-Xa activity in these samples. Therefore, anti-Xa analysis may be used instead of aPTT or ACT to guide heparin therapy in PwHA treated with emicizumab.

The molecular mechanism of emicizumab clearly interacts with the underlying principles of these assay groups in a predictable way, and results from this study can be used by clinicians to inform the selection of appropriate assays when monitoring haemostasis in PwHA receiving emicizumab therapy. According to our findings, the interference potential of emicizumab on one-stage aPTT-based assays may be mitigated by using chromogenic- or immunogenic-based methodology. Specifically, the interference potential of emicizumab on FVIII one-stage assays may be mitigated by using a two-stage chromogenic assay, provided it contains human FX and FIXa, such as the BIOPHEN FVIII:C assay by HYPHEN BioMed. The chromogenic FVIII assay using human FIXa and FX was responsive to emicizumab. Unlike the one-stage FVIII assay, endogenous FVIII in the plasma sample is preactivated by thrombin in this chromogenic assay, which reduces the difference in relative potency between FVIII and emicizumab. In the clinically relevant emicizumab plasma concentration range of approximately 25 to $80 \mu \mathrm{g} / \mathrm{mL}^{4}{ }^{4}$ the relationship between emicizumab concentration and reported FVIII activity was almost linear, with reported FVIII activities of approximately 20 to $50 \%$, consistent with the linear relationship reported in clinical data. ${ }^{10,25}$

However, FVIII activity reported by this test for an emicizumab patient should not be viewed as equivalent to FVIII activity data obtained from FVIII-treated patients because emicizumab and FVIII have different enzymatic properties in relation to the tenase reaction. ${ }^{3,26}$ Furthermore, FVIIIa is rapidly inactivated by spontaneous dissociation of the A2 subunit as well as by proteolysis (reviewed in Fay) ${ }^{27}$ while emicizumab remains active, and the differences in binding affinity to FXIa and FX indicate a probable lack of parallelism at different concentrations of these factors. Therefore, care should be taken in using the BIOPHEN assay outside of the roughly linear range of approximately 25 to $80 \mu \mathrm{g} / \mathrm{mL}$ emicizumab, or if using human FVIII chromogenic assays other than the single kit tested here. Nevertheless, the human FVIII chromogenic assay can provide a relative indication of the procoagulant activity of emicizumab. In contrast to the human chromogenic FVIII assay, chromogenic FVIII assays that contain bovine FX and FIXa are nonresponsive to emicizumab due to the species specificity of emicizumab for human FIXa and FX. Most chromogenic FVIII assays currently on the market, such as the assays by Stago, Siemens, and Instrumentation Laboratory, use bovine FX and FIXa. These assays allow for measurement of endogenous or infused FVIII activity without interference in PwHA receiving emicizumab treatment. Therefore, they may be used to monitor FVIII concentrations in the 
presence of emicizumab or as the basis for detection of FVIII inhibitors using the chromogenic Bethesda assay, as explained below. $^{28}$

Differences in activity measured by one-stage compared with chromogenic FVIII assays is a frequent finding for the extended half-life FVIII-derived therapeutics approved or in development for haemophilia A, including B-domain deleted, PEGylated, and single-chain FVIII molecules. Many of these molecules show selective effects in one-stage assays depending on the type of aPTT reagent used (reviewed in Kitchen et al), ${ }^{29}$ making clinical interpretation challenging and leading to calls for product-specific reference standards. ${ }^{30,31}$ In

Table 2 Assays not affected by emicizumab

\begin{tabular}{|c|c|c|}
\hline Assay & Principle & Activator \\
\hline $\begin{array}{l}\text { Fibrinogen according } \\
\text { to Clauss }\end{array}$ & \multirow[t]{3}{*}{$\begin{array}{l}\text { Clotting } \\
\text { assay }\end{array}$} & \multirow[t]{2}{*}{ Thrombin } \\
\hline Thrombin time & & \\
\hline $\begin{array}{l}\text { Prothrombin } \\
\text { activator-based } \\
\text { APC resistance test }\end{array}$ & & $\begin{array}{l}\text { Prothrombin } \\
\text { activator }\end{array}$ \\
\hline Anti-Xa activity & \multirow{4}{*}{$\begin{array}{l}\text { Amidolytic } \\
\text { assay }\end{array}$} & FXa \\
\hline $\begin{array}{l}\text { Protein C chromogenic } \\
\text { assay }\end{array}$ & & Protein $\mathrm{C}$ activator \\
\hline Antithrombin activity & & Thrombin \\
\hline Plasminogen activity & & Streptokinase \\
\hline Plasminogen antigen & ELISA & \multirow[t]{6}{*}{ Not applicable } \\
\hline Free protein $\mathrm{S}$ antigen & \multirow{5}{*}{$\begin{array}{l}\text { Latex } \\
\text { assay }\end{array}$} & \\
\hline D-dimer concentration & & \\
\hline vWF antigen & & \\
\hline vWF activity & & \\
\hline FXIII antigen & & \\
\hline
\end{tabular}

Abbreviations: APC, activated protein C; ELISA, enzyme-linked immunosorbent assay; FXa, activated factor X; FXIII, factor XIII; vWF, von Willebrand factor. contrast, the interference effects of emicizumab are mechanism-based and therefore universal: 13 different types of aPTT reagents exhibited a similar overshortening effect with emicizumab. ${ }^{32}$ Indeed, several medical groups have issued guidance that all one-stage FVIII assays should be avoided, irrespective of reagent type, for persons receiving emicizumab therapy. ${ }^{33,34}$ As a possible alternative, chromogenic FVIII assays may be used either to measure relative emicizumab effect (human reagents; e.g., HYPHEN BioMed kit) or to measure endogenous/infused FVIII (bovine reagents; most other commercial kits).

Importantly, the interference effect of emicizumab in onestage FVIII assays also results in false negative measurements of FVIII inhibitors when using the classic Bethesda or Nijmegen-Bethesda assays, even with a heat-inactivation step. Alternative tests include a chromogenic Bethesda assay ${ }^{28}$ that employs a bovine-based chromogenic FVIII activity readout (insensitive to emicizumab), or the addition of anti-emicizumab reagent antibodies to the plasma sample to neutralise the drug prior to testing. ${ }^{35} \mathrm{~A}$ chromogenic Bethesda assay has been deployed successfully to measure FVIII inhibitor titers in clinical trials of emicizumab ${ }^{25}$ and will be addressed in detail in a future manuscript.

One limitation of this exploratory study is that it included plasma samples from only a small number of individual donors that were spiked with emicizumab, and not samples from individuals receiving emicizumab therapy. However, plasma samples were spiked to produce emicizumab concentrations below, across, and above the therapeutic range, allowing for comprehensive profiling of interference effects, and could therefore be extrapolated to the clinical setting. The concordance in concentration-effect relationship between in vitro results reported here and clinical values for aPTT, FVIII one-stage, and FVIII chromogenic assays further support the validity of the extrapolation for the other assays. Only one reagent was tested for each assay, with the exception of the PT assay (four reagents tested); however, several different reagents are commercially available for most of the assays evaluated here, and it is possible that

Table 3 Assays affected by emicizumab, with mitigation options

\begin{tabular}{|c|c|c|c|}
\hline Assay & Principle & Activator & Mitigation \\
\hline aPTT & \multirow[t]{5}{*}{$\begin{array}{l}\text { Clotting } \\
\text { (chronometric) }\end{array}$} & $\begin{array}{l}\text { Contact activator } \\
\text { (kaolin, silica, etc.) }\end{array}$ & For heparin monitoring: anti-Xa assay \\
\hline $\begin{array}{l}\text { aPTT-based } \\
\text { protein } C \text { assay }\end{array}$ & & Protein $\mathrm{C}$ activator & Chromogenic protein $\mathrm{C}$ assay \\
\hline $\begin{array}{l}\text { aPTT-based } \\
\text { protein S assay }\end{array}$ & & $\begin{array}{l}\text { Contact activator/ } \\
\text { APC/FVa }\end{array}$ & Free protein $\mathrm{S}$ assay \\
\hline $\begin{array}{l}\text { aPTT-based APC } \\
\text { resistance assay }\end{array}$ & & $\begin{array}{l}\text { Contact activator/ } \\
\text { APC }\end{array}$ & $\begin{array}{l}\text { Prothrombin activator-based test for APC resistance, } \\
\text { gene test for FV Leiden mutation }\end{array}$ \\
\hline PT (weak effect) & & \multirow[t]{2}{*}{ TF } & $\begin{array}{l}\text { No mitigation required (small effect); PT reagent selection } \\
\text { also will mitigate }\end{array}$ \\
\hline $\begin{array}{l}\text { Derived fibrinogen } \\
\text { (weak effect) }\end{array}$ & $\begin{array}{l}\text { Clotting } \\
\text { (photometric) }\end{array}$ & & $\begin{array}{l}\text { No mitigation required (small effect); also Clauss fibrinogen is } \\
\text { unaffected by emicizumab }\end{array}$ \\
\hline
\end{tabular}

Abbreviations: APC, activated protein C; aPTT, activated partial thromboplastin time; FVa, activated factor V; FXa; activated factor X; PT, prothrombin time; TF, tissue factor. 
emicizumab would have differential effects on assay outcomes using these systems. Additionally, many more coagulation assays are available other than those evaluated in our present study; therefore, further studies are required to fully elucidate the effect of emicizumab on all coagulation assays used in clinical practice.

In conclusion, based on its mechanism of action, emicizumab interferes with all aPTT-based assays tested, but not with assays based on immunologic or chromogenic principles, nor with clotting assays based on nonintrinsic pathway activators. Alternative testing strategies are available as summarised in - Tables 2 and 3. In particular, for PwHA receiving emicizumab therapy, FVIII activity should not be measured with a one-stage assay, but may be measured using bovine chromogenic assays, while emicizumab activity may be measured using human chromogenic assays, keeping in mind the caveats and limitations described above. A modified one-stage FVIII assay, which uses a dedicated calibrator and controls for emicizumab, is also in development for the measurement of emicizumab plasma concentration. ${ }^{36}$ Potential assay interference effects should always be considered in the selection and interpretation of test results for PwHA receiving emicizumab treatment.

\section{What is known about this topic?}

- Strong pharmacodynamic effects of emicizumab on activated partial thromboplastin time, chromogenic factor VIII assays using human factor X (FX)/activated factor IX (FIXa), rotational thromboelastometry, and thrombin generation testing have been reported. However, the effect of emicizumab on additional coagulation assays in routine clinical use has not been evaluated thus far.

\section{What does this paper add?}

- The current work summarises the effects of emicizumab on commonly used assays and identifies coagulation assays that may be used in persons with hemophilia A receiving treatment with emicizumab, and available alternatives that are suitable to replace assays that exhibit strong interference by emicizumab.

\section{Note}

Investigations were performed at Menal GmbH, Emmendingen, Germany.

\section{Funding}

This work was financially supported by F. Hoffmann-La Roche, Ltd.

\section{Conflict of Interest}

Joanne I. Adamkewicz, David C. Chen, and Ido Paz-Priel are employees of Genentech, Inc.

\section{Acknowledgments}

This study was sponsored by F. Hoffmann-La Roche Ltd. We thank Andreas Calatzis, PhD, for his contribution in the development of prior versions of this manuscript. Writing assistance for this manuscript was provided by Leon Newman, PhD, Gardiner-Caldwell Communications, and was funded by F. Hoffmann-La Roche Ltd.

\section{References}

1 Kitazawa T, Igawa T, Sampei Z, et al. A bispecific antibody to factors IXa and $X$ restores factor VIII hemostatic activity in a hemophilia A model. Nat Med 2012;18(10):1570-1574

2 Sampei Z, Igawa T, Soeda T, et al. Identification and multidimensional optimization of an asymmetric bispecific $\operatorname{IgG}$ antibody mimicking the function of factor VIII cofactor activity. PLoS One 2013;8(02):e57479

3 Kitazawa T, Esaki K, Tachibana T, et al. Factor VIIla-mimetic cofactor activity of a bispecific antibody to factors IX/IXa and $\mathrm{X} / \mathrm{Xa}$, emicizumab, depends on its ability to bridge the antigens. Thromb Haemost 2017;117(07):1348-1357

4 Oldenburg J, Mahlangu JN, Kim B, et al. Emicizumab prophylaxis in hemophilia A with inhibitors. N Engl J Med 2017;377(09):809-818

5 Young G, Liesner R, Sidonio RF, et al. Emicizumab prophylaxis provides flexible and effective bleed control in children with hemophilia A with inhibitors: results from the HAVEN 2 Study. Blood 2018;132(Suppl 1):632

6 Mahlangu J, Oldenburg J, Paz-Priel I, et al. Emicizumab prophylaxis in patients who have hemophilia A without inhibitors. N Engl J Med 2018;379(09):811-822

7 Pipe SW, Shima M, Lehle M, et al. Efficacy, safety, and pharmacokinetics of emicizumab prophylaxis given every 4 weeks in people with haemophilia A (HAVEN 4): a multicentre, open-label, non-randomised phase 3 study. Lancet Haematol 2019. Doi: 10.1016/S2352-3026(19)30054-7

8 Lawson JH, Kalafatis M, Stram S, Mann KG. A model for the tissue factor pathway to thrombin. I. An empirical study. J Biol Chem 1994;269(37):23357-23366

9 Uchida N, Sambe T, Yoneyama K, et al. A first-in-human phase 1 study of ACE910, a novel factor VIII-mimetic bispecific antibody, in healthy subjects. Blood 2016;127(13):1633-1641

10 Shima M, Hanabusa H, Taki M, et al. Long-term safety and efficacy of emicizumab in a phase $1 / 2$ study in patients with hemophilia $A$ with or without inhibitors. Blood Adv 2017;1(22):1891-1899

11 Shima M, Hanabusa H, Taki M, et al. Factor VIII-mimetic function of humanized bispecific antibody in hemophilia A. N Engl J Med 2016;374(21):2044-2053

12 Al-Samkari H, Croteau SE. Shifting landscape of hemophilia therapy: implications for current clinical laboratory coagulation assays. Am J Hematol 2018. Doi: 10.1002/ajh.25153

13 Srivastava A, Brewer AK, Mauser-Bunschoten EP, et al; Treatment Guidelines Working Group on behalf of the World Federation of Hemophilia. Guidelines for the management of hemophilia. Haemophilia 2013;19(01):e1-e47

14 F. Hoffmann-La Roche Ltd. Summary of Product Characteristics Hemlibra: EMA; 2018 Available at: http://www.ema.europa.eu/docs/ en_GB/document_library/EPAR_-_Product_Information/human/004 406/WC500244743.pdf. Accessed April 14, 2019

15 Adamkewicz J, Schmitt C, Asikanius E, et al. Factor VIII inhibitor testing using a validated chromogenic Bethesda assay in HAVEN 1 (BH29884), a phase 3 trial of emicizumab in persons with hemophilia A with inhibitors. Res Pract Thromb Haemost 2017;1(Suppl 1):724

16 Duncan E, Rodgers S. One-stage factor VIII assays. Methods Mol Biol 2017; 1646:247-263

17 Riley PW, Gallea B, Valcour A. Development and implementation of a coagulation factor testing method utilizing autoverification in 
a high-volume clinical reference laboratory environment. J Pathol Inform 2017;8:25

18 Kitchen S, Signer-Romero K, Key NS. Current laboratory practices in the diagnosis and management of haemophilia: a global assessment. Haemophilia 2015;21(04):550-557

19 Palareti G, Maccaferri M. Specific assays of hemostasis proteins: fibrinogen. Ric Clin Lab 1990;20(02):167-176

20 Barriere SL, Goldberg MR, Janc JW, Higgins DL, Macy PA, Adcock DM. Effects of telavancin on coagulation test results. Int J Clin Pract 2011;65(07):784-789

21 Hylek EM, Singer DE. Risk factors for intracranial hemorrhage in outpatients taking warfarin. Ann Intern Med 1994;120(11): 897-902

22 Hylek EM, Go AS, Chang Y, et al. Effect of intensity of oral anticoagulation on stroke severity and mortality in atrial fibrillation. N Engl J Med 2003;349(11):1019-1026

23 Fang MC, Chang Y, Hylek EM, et al. Advanced age, anticoagulation intensity, and risk for intracranial hemorrhage among patients taking warfarin for atrial fibrillation. Ann Intern Med 2004;141 (10):745-752

24 Hattersley PG. Progress report: the activated coagulation time of whole blood (ACT). Am J Clin Pathol 1976;66(05):899-904

25 Adamkewicz J, Schmitt C, Asikanius E, et al. Factor VIII inhibitor testing using a validated chromogenic Bethesda assay (CBA) in HAVEN 1 (BH29884), a phase 3 trial of emicizumab in persons with hemophilia A (PwHA) with inhibitors. ISTH 2017; poster 954. Available at: https://www.postersessiononline.eu/173580348_eu/ congresos/ISTH2017/aula/-PB_954_ISTH2017.pdf.Accessed April 14, 2019

26 Lenting PJ, Denis CV, Christophe OD. Emicizumab, a bispecific antibody recognizing coagulation factors IX and X: how does it actually compare to factor VIII? Blood 2017;130(23):2463-2468

27 Fay PJ. Factor VIII structure and function. Int J Hematol 2006;83 (02):103-108

28 Miller CH, Rice AS, Boylan B, et al; Hemophilia Inhibitor Research Study Investigators. Comparison of clot-based, chromogenic and fluorescence assays for measurement of factor VIII inhibitors in the US Hemophilia Inhibitor Research Study. J Thromb Haemost 2013;11(07):1300-1309

29 Kitchen S, Kershaw G, Tiefenbacher S. Recombinant to modified factor VIII and factor IX - chromogenic and one-stage assays issues. Haemophilia 2016;22(Suppl 5):72-77

30 Bulla O, Poncet A, Alberio L, et al. Impact of a product-specific reference standard for the measurement of a PEGylated rFVIII activity: the Swiss Multicentre Field Study. Haemophilia 2017;23 (04):e335-e339

31 Ingerslev J, Jankowski MA, Weston SB, Charles LA; ReFacto Field Study Participants. Collaborative field study on the utility of a BDD factor VIII concentrate standard in the estimation of BDDr Factor VIII:C activity in hemophilic plasma using one-stage clotting assays. J Thromb Haemost 2004;2(04):623-628

32 Adamkewicz J, Kim B, Calatzis A. Measurement of FVIII inhibitor titer using a Chromogenic Bethesda Assay (CBA) in the presence of emicizumab (ACE910), a humanized bispecific antibody mimicking FVIIla cofactor function. Haemophilia 2017;23(Suppl 3):3

33 Collins PW, Liesner R, Makris M, et al. Treatment of bleeding episodes in haemophilia A complicated by a factor VIII inhibitor in patients receiving emicizumab. Interim guidance from UKHCDO Inhibitor Working Party and Executive Committee. Haemophilia 2018;24(03):344-347

34 Medical and Scientific Advisory Council (MASAC) of the National Hemophilia Foundation (NHF). Recommendation on the use and management of emicizumab-kxwh (HEMLIBRA ${ }^{\circledR}$ ) for hemophilia A with and without inhibitors (MASAC Document \#255); December 2018. Available at: https://www.hemophilia.org/sites/default/files/ document/files/255Emicizumab.pdf. Accessed April 14, 2019

35 Nogami K, Soeda T, Matsumoto T, Kawabe Y, Kitazawa T, Shima M. Routine measurements of factor VIII activity and inhibitor titer in the presence of emicizumab utilizing anti-idiotype monoclonal antibodies. J Thromb Haemost 2018;16(07):1383-1390

36 Calatzis A, McInerney M, Calhoon W, Adamkewicz J, Morris M, Chen D. Quantification of emicizumab based on a modified onestage FVIII assay. Paper presented at: Annual Meeting of the Society of Thrombosis and Hemostasis Research; 2018 Vienna 